\title{
Spectrum allocation and mode selection for overlay D2D using carrier sensing threshold
}

\author{
Byungjin Cho, Konstantinos Koufos and Riku Jäntti \\ Communications and Networking Department, Aalto University, Finland \\ Byungjin.Cho@aalto.fi, Konstantinos.Koufos@aalto.fi, Riku.Jantti@aalto.fi
}

\begin{abstract}
In this paper, we propose a mechanism for mode selection and spectrum allocation for in-band overlay D2D communication. A potential D2D user measures the activity over the spectrum allocated for D2D transmissions and uses a carrier sensing threshold to decide about its transmission mode. By appropriately selecting the carrier sensing threshold, the interference among D2D communication pairs can be controlled and their performance can be improved. Also, the distributed nature of this mechanism leads to less signalling overhead between D2D users and base stations even in dense deployments. Based on this method, we find spectrum allocation factors and carrier sensing thresholds for maximizing the rate of D2D users under target rate constraint for cellular users.
\end{abstract}

Index Terms-Device-to-device communication, Matérn point process, mode selection, spectrum sharing, stochastic geometry.

\section{INTRODUCTION}

Device-to-Device (D2D) communication in cellular spectrum is a promising concept holding potential benefits such as improved spectrum efficiency, enhanced capacity and improved coverage [1]-[3]. One fundamental issue in supporting D2D in cellular networks is how to efficiently utilize the cellular spectrum resources. In terms of conceptual and theoretical model, D2D can be seen as an extension of the concept of cognitive radio and dynamic spectrum access, where the cellular links can be seen as primary links and the D2D as secondary links. The difference with the cognitive radio model is that in D2D communication the primary system is aware and supportive about the secondary links. Under appropriate control of the base station (BS), D2D can improve the spectrum efficiency of cellular networks by reusing cellular resources (D2D underlay) or allocating dedicated cellular resources for D2D communication (D2D overlay).

In the underlay approach, interference issue arises since D2D and cellular users on the same resource can cause severe interference to each other. Thus, proper resource allocation should be performed. Previous works, see for instance [2], [3], assumed that BS knows the channel state information of all involved links. The BS's participation to make scheduling decision for cellular and D2D users causes large signalling overhead especially in dense deployments.

On the other hand, in overlay approach, there is no crosstier interference between cellular and D2D users [2]. However, the cellular spectrum might be used inefficiently. One way to improve spectrum utilization is to use proper mode selection algorithms which would determine the actual density of D2D transmissions in the network.

In the existing literature, mode selection algorithms utilize either the D2D pair distance [4] and/or the distance between D2D transmitter and cellular BS [5] as selection criteria. In that case, a D2D transmitter can generate harmful interference to another D2D communication pair, as D2D pairs can be arbitrary close to each other.

In this paper, we consider overlay D2D and schedule the transmissions of D2D users so that the transmissions in D2D mode are placed at least at some distance far away from each other. In this way, the self-interference among the D2D pairs can be controlled. In order to select the mode, we propose that a D2D user should measure the activity over the spectrum allocated for transmissions in D2D mode and use a carrier sensing (CS) threshold to decide about its transmission mode. When the measured energy is below the threshold, there is indication there are not many ongoing D2D communications and D2D mode should be selected. Otherwise, the D2D user should select infrastructure-based mode. As a result, mode selection is done in a distributed fashion eliminating signalling overhead between BS and D2D users.

Considering the system performance, the CS threshold and the amount of cellular spectrum allocated exclusively for D2D communication are coupled. For instance, in dense deployments, a high CS threshold results in high D2D density and subsequently in high interference among D2D communication pairs. This can be compensated by allocating to them more spectrum. However, this means there would be less spectrum available for cellular transmissions. In this paper, we show how to set the CS threshold and the spectrum partition factor for maximizing the rate of D2D users under target rate constraint for the cellular users.

\section{SySTEM MODEL AND MODE SELECTION}

We consider a cellular system that enables D2D communication. The cellular users are distributed according to a Poisson point process (PPP), $\Phi_{c}$, with density $\lambda_{c}$ while D2D users follow a PPP, $\Phi$, with density $\lambda$. The density of base stations (BSs) is denoted by $\lambda_{b s}$ and their distribution follows a PPP too. The BSs form a Voronoi tessellation and cellular users communicate with their nearest BS (also referred to as home BS). On the other hand, D2D users may use either 
D2D communication mode or infrastructure-based mode (also referred to as cellular-based mode) through their home BS.

We consider overlay in-band D2D where a fraction $\beta$ of the cellular spectrum is exclusively assigned for $\mathrm{D} 2 \mathrm{D}$ mode. The remaining part $(1-\beta)$ is used for cellular-based transmissions, see Fig. 1. Within a macro-cell, the cellular users and the D2D users in cellular mode are scheduled in a round-robin fashion i.e. only a single user can be active at a time and it uses the full available bandwidth $(1-\beta)$. On the other hand, there may be many ongoing D2D communication pairs within a macro-cell each using bandwidth $\beta$.

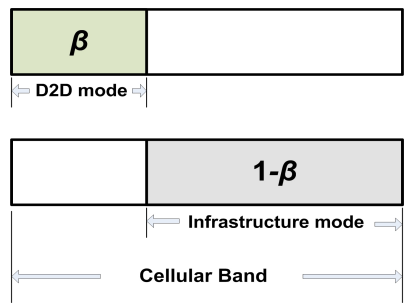

Fig. 1: In-band overlay D2D. Portion $\beta$ of cellular spectrum is exclusively allocated for D2D communication mode.

The D2D pair distance is fixed and denoted by $d$ while the transmit power level in D2D mode is equal to $P_{d}$. For a small distance $d$, the power level $P_{d}$ should be taken much smaller than the power level $P$ used for cellular transmissions. For the time being, we do not incorporate power control in our analysis neither for cellular nor for D2D users. The distancebased propagation pathloss as a function of the distance $r$ is denoted by $l_{d}(r)$ for D2D mode and $l_{c}(r)$ for cellular mode. Also, we consider Rayleigh fading with mean equal to unity.

In underlay D2D, the D2D communication is usually allocated over uplink resources to better control the interference the D2D mode generates to the cellular system. Without impairments such as out-of-band emissions and adjacent channel frequency selectivity, there are no similar restrictions in overlay D2D. However, we still present our analysis for an uplink cellular system as we plan to conduct a future study on the performance of overlay D2D in the presence of impairments.

\section{A. Mode selection}

In order to select the mode for the D2D users we consider their mutual interference. We try to avoid situations where D2D communication pairs located close to each other are all scheduled in D2D mode. At the same time, we are looking for mechanisms with a low communication signalling overhead between the D2D users and their home BS.

In order to fulfill these requirements, we propose that D2D users could measure the activity in the D2D spectrum and use a threshold-based test to decide their mode in a distributed way. When the measured energy is below the threshold, there is indication that there are not many ongoing D2D communications closeby and D2D mode is selected. Otherwise, the D2D users select infrastructure-based mode. Essentially, D2D users employ a carrier sensing multiple access (CSMA) type of contention to transmit in D2D mode. Provided that the D2D pair distance $d$ is smaller than the CS range, the D2D selfinterference can be controlled. In Fig. 2, one can see that potential D2D users inside the CS range of an ongoing D2D communication resort to infrastructure-based mode.

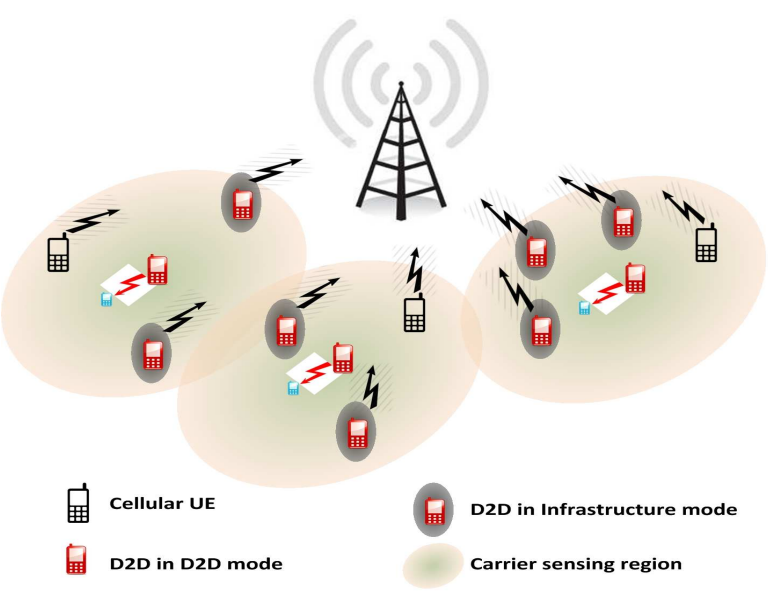

Fig. 2: Illustration of the mode selection algorithm.

\section{B. Carrier sensing range and carrier sensing threshold}

The locations of transmitters in wireless networks with contention control are usually modelled by the Matérn Point Process (MPP) [6]. Even though MPP type II suffers from the well-known node underestimation problem, it has been widely employed to model the distribution of transmitters in wireless networks with CSMA type of contention control [7], [8]. The set of D2D users scheduled in D2D mode, $\Phi_{d}$, can be obtained by thinning the parent PPP, $\Phi$, and the density of transmissions in D2D mode, $\lambda_{d}$, is equal to the density of an MPP type II with PPP parent density $\lambda$ and hardcore distance (HCD) $\delta$,

$$
\lambda_{d}=\frac{1-e^{-\lambda \pi \delta^{2}}}{\pi \delta^{2}} .
$$

Equivalently, a D2D user transmits in D2D mode with probability $q=\lambda_{d} / \lambda$ and in cellular mode with complementary probability $(1-q)$.

The HCD $\delta$ models the CS range. Given the HCD, the mean interference at a typical D2D transmitter can be computed and set equal to the CS threshold [8] which would be used in practice to control the density of D2D mode transmissions.

According to the properties of MPP type II, the mean interference at the D2D transmitter can be split into two terms: (i) The mean interference from D2D transmitters located at distances $r>2 \delta$ is equal to the mean interference due to a PPP with density $\lambda_{d}$. (ii) The mean interference due to transmissions at distances $r: \delta \leq r \leq 2 \delta$ depends on the correlation properties of the MPP type II. The pair correlation function (PCF) for MPP type II has a complex form but simple bounds have been derived in [9] and can be used to approximate the relation between HCD and CS threshold. 
Note that an upper bound for the CS threshold will result in less D2D users allocated in cellular spectrum and thus, it favors the QoS of cellular users. Using the upper bound of the PCF, we obtain the following upper bound for the CS threshold

$$
\begin{aligned}
E_{o}^{!}\left\{I_{t}\right\} & =E_{o}^{!}\left\{I_{t_{<2 \delta}}\right\}+E_{o}^{!}\left\{I_{t_{>2 \delta}}\right\} \\
& \leq 2 \pi \lambda_{d} \int_{\delta}^{2 \delta} l_{d}(r) \bar{g}(r) d r+2 \pi \lambda_{d} \int_{2 \delta}^{\infty} l_{d}(r) d r
\end{aligned}
$$

where $I_{t}$ denotes the interference level at the D2D transmitter and $\bar{g}(r)$ is the upper bound for the PCF of MPP type II. Also, note that the mean of the fading which is equal to unity has been omitted from the equation.

\section{SPECTRUM ALLOCATION OPTIMIZATION}

Given the densities of the D2D users, BSs and cellular users, a network management entity should divide the spectrum between D2D and infrastructure communication and also set the CS threshold for the D2D users. These values are then broadcasted from the BSs to the D2D users.

\section{A. Problem formulation}

The CS threshold and the spectrum partition factor can be selected to maximize various optimization criteria of the cellular system. In this paper, we propose to set the optimization parameters for maximizing the rate of the D2D users, $Q_{d}$, under some constraint on the rate in the cellular uplink, $Q_{c}$.

$$
\begin{array}{cc}
\underset{\beta}{\operatorname{Maximize}}: & Q_{d} . \\
\text { Subject to : } & Q_{c} \geq \tau .
\end{array}
$$

Next we show how to compute $Q_{d}$ and $Q_{c}$. First, let us consider a typical D2D link. With overlay in-band D2D, the interferers are D2D transmitters. The interference at the typical D2D receiver is given by $I_{d}=\sum_{x \in \Phi_{d}} P_{d} h_{x} l_{d}\left(r_{x}\right)$ where $h_{x}$ describes the fading from interferer $x$ and follows exponential distribution with mean equal to unity. Note that the probability generating functional (PGFL) of MPP type II is not known. As a result, only an approximation for the coverage probability of transmissions in D2D mode can be obtained. In Appendix A, the following approximation has been obtained

$$
\mathcal{P}_{d} \approx e^{-\frac{\gamma_{d} \cdot \beta \sigma_{d}^{2}}{P_{d} \cdot l_{d}(d)}-\lambda_{d}} \int_{0}^{2 \pi} \int_{2 \delta}^{\infty} \frac{f(r)}{1+f(r)} r d r d \phi-c \lambda_{d} \int_{0}^{2 \pi} \int_{\delta}^{2 \delta} \frac{f(r)}{1+f(r)} r d r d \phi
$$

where $\gamma_{d}$ is the SINR target, $\sigma_{d}^{2}$ is the noise level at D2D receiver over the full bandwidth, the constant $c=\frac{2 \pi}{4 \pi / 3+\sqrt{3} / 2}$ and $f(r)=\gamma_{d} \cdot l\left(\sqrt{r^{2}+d^{2}-2 r d \cos \phi}\right) / l(d)$.

Using the above approximation, the spectral efficiency for D2D mode can be derived based on [4]

$$
R_{d}=\int_{0}^{\infty} \frac{\mathcal{P}_{d}}{1+\gamma_{d}} d \gamma_{d}
$$

Let us now consider a typical cellular link. With overlay inband $\mathrm{D} 2 \mathrm{D}$, the interferers are cellular transmitters from other cells i.e. cellular users and D2D users in cellular mode. Recall from Section II that cellular users are distributed according to a PPP with density $\lambda_{c}$. Also, D2D users in cellular mode would generate in the uplink mean interference equal to the mean interference from a PPP with density $(1-q) \lambda$. As these two PPPs are independent, one can approximate their aggregate impact by another PPP, $\Phi_{c}^{\prime}$, with density $\lambda_{c}^{\prime}=\lambda_{c}+(1-q) \lambda[6]$.

In the uplink of a cellular system with round-robin scheduling, only one transmitter is active in a cell at particular moment. Scheduling introduces dependency in the process $\Phi_{c}^{\prime}$. To simplify the analysis, we assume that the typical active cellular user is uniformly distributed in the cell area of a BS and the locations of cellular interferers form a PPP $\Phi_{c, a}^{\prime}$ with density $\lambda_{b s}$. Under this assumption, it can also be shown that the distance of a cellular user to its nearest BS follows the Rayleigh distribution $h_{R}(r)=2 \pi \lambda_{b s} r e^{-\lambda_{b s} \pi r^{2}} \quad$ [4].

The interference at the typical BS is given by $I_{c}=$ $\sum_{y \in \Phi_{c, a}^{\prime}} P_{c} h_{y} l_{c}\left(r_{y}\right)$ where $h_{y}$ describes the fading from the $y$-th interferer following exponential distribution with mean equal to unity. While computing coverage probability for cellular transmissions, one should take into account the fact that some BSs may not have any user to serve.

Lemma 1. The coverage probability for cellular users is

$$
\mathcal{P}_{c}=\int_{0}^{\infty} h_{R}(r) \cdot e^{-\frac{\gamma_{c}(1-\beta) \sigma_{c}^{2}}{P_{c} \cdot l(r)}-2 \pi \alpha \lambda_{b s} \frac{r^{2} \gamma_{c}}{a-2} 2 F_{1}\left(1, \frac{a-2}{a}, 2-\frac{2}{a},-\gamma_{c}\right)} d r
$$

where $\gamma_{c}$ is the cellular SINR target, $\sigma_{c}^{2}$ is the noise power level at the base station over the full bandwidth, $\alpha=1-$ $\left(1+3.5^{-1} \lambda_{c}^{\prime} / \lambda_{b s}\right)^{-3.5}$ is the probability a base station is active [10] and ${ }_{2} F_{1}$ is the Gaussian hypergeometric function.

\section{Proof: See Appendix B.}

Based on Lemma 1, the spectral efficiency in the cellular uplink can be derived as [4]

$$
R_{c}=\nu \int_{0}^{\infty} \frac{\mathcal{P}_{c}}{1+\gamma_{c}} d \gamma_{c}
$$

where $\nu=\alpha \lambda_{b s} / \lambda_{c}^{\prime}$ is the portion of time a cellular user is active in the uplink.

Using the link rates $R_{c}$ and $R_{d}$, we can derive the average rates of cellular and D2D users, $Q_{c}$ and $Q_{d}$ respectively. The average rate of cellular users, $Q_{c}$, is equal to their spectral efficiency, $R_{c}$, multiplied by the available bandwidth, (1 $\beta$ ). On the other hand, the average rate of D2D users, $Q_{d}$, is obtained as an average of their normalized spectral efficiency in D2D mode, $R_{d}$, and cellular mode, $R_{c}$, scaled with the normalized user density and transmission bandwidth.

Proposition 1. The average normalized rate for cellular and D2D users is

$$
\begin{aligned}
& Q_{c}=(1-\beta) R_{c} \\
& Q_{d}=q \beta R_{d}+(1-q)(1-\beta) R_{c} .
\end{aligned}
$$




\section{B. Optimization algorithm}

We start by showing how to identify the feasibility region i.e. CS thresholds and spectrum allocation factors that satisfy the optimization constraint $(1-\beta) R_{c} \geq \tau$. In general, the spectral efficiency $R_{c}$ depends on both optimization parameters making the numerical analysis complex. To simplify matters, we propose to ignore the impact of noise level on the coverage probability of cellular transmissions i.e. we set $\sigma_{c}^{2}=0$ in equation (6). The proposed approximation is accurate in dense deployments, see Fig. 3, because the cellular system becomes interference limited.

Given a CS threshold or equivalently a $\operatorname{HCD} \delta$, the spectral efficiency $R_{c}$ is obtained after a double numerical integration, see equation (6), and the largest possible spectrum allocation factor is expressed as $\beta \leq 1-\frac{\tau}{R}$. With the feasibility region at hand, the $\mathrm{D} 2 \mathrm{D}$ rate $Q_{d}=q \beta R_{d}+(1-q) \tau$ is evaluated for different pairs of values for the HCD and the spectrum allocation factor $\beta$ along the border of the feasibility region. It is a matter of future research to prove that the maximum of the $\mathrm{D} 2 \mathrm{D}$ rate $Q_{d}$ occurs on the feasibility border.

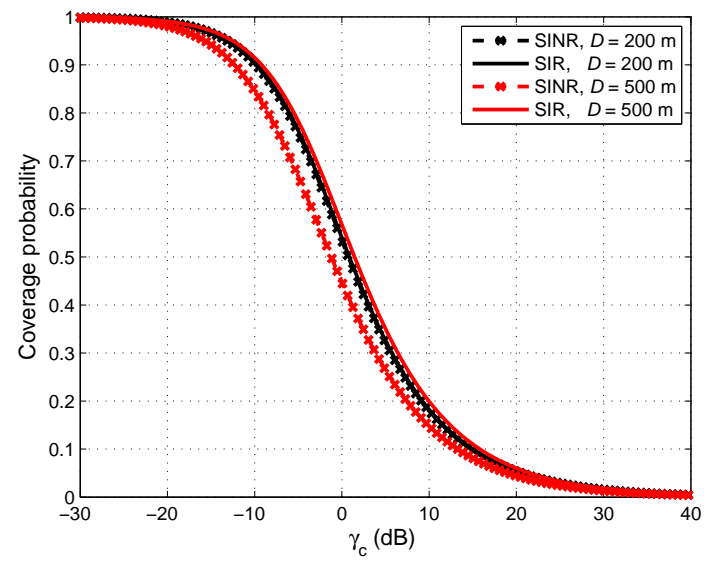

Fig. 3: Coverage probability based on SIR and SINR in cellular uplink for different cell radiuses $D$. The noise level has been taken equal to $-96 \mathrm{dBm}$ assuming that full $20 \mathrm{MHz}$ band is allocated to cellular mode. The noise figure is $5 \mathrm{~dB}$.

\section{NUMERICAL RESULTS}

We consider a cellular network with BS density $\lambda_{b s}=$ $1 /\left(\pi \cdot 200^{2}\right)$ and cellular users with twice the BS density, $\lambda_{c}=2 \lambda_{b s}$. We evaluate the performance of spectrum allocation and mode selection algorithm for different D2D user densities $\lambda=\{1 \cdots 10\} \times \lambda_{b s}$. Taking realistic 3GPP propagation environment [11] into account, we consider the following pathloss equation in $\mathrm{dB}: 37.6 \log _{10}(r)+15.3$ for the infrastructure mode and $40.0 \log _{10}(r)+28$ for the D2D mode, where $r$ is the distance in meters. The D2D link distance is fixed to $d=30 \mathrm{~m}$. We use fixed transmit power level, $23 \mathrm{dBm}$ for the infrastructure mode and $20 \mathrm{dBm}$ for the D2D mode.

In Fig. 4 we depict the feasibility region i.e. CS thresholds and spectrum allocation factors $\beta$ given the constraint $\tau$ on the normalized cellular rate. In general, increasing the constraint $\tau$ reduces the size of the feasibility region for D2D mode because more bandwidth should be reserved for cellular-based communication. Also, given a rate constraint $\tau$, allocating more spectrum for the D2D mode should always be combined with a higher CS threshold to increase the density of users in D2D mode and leave enough time resources available for cellularbased communication.

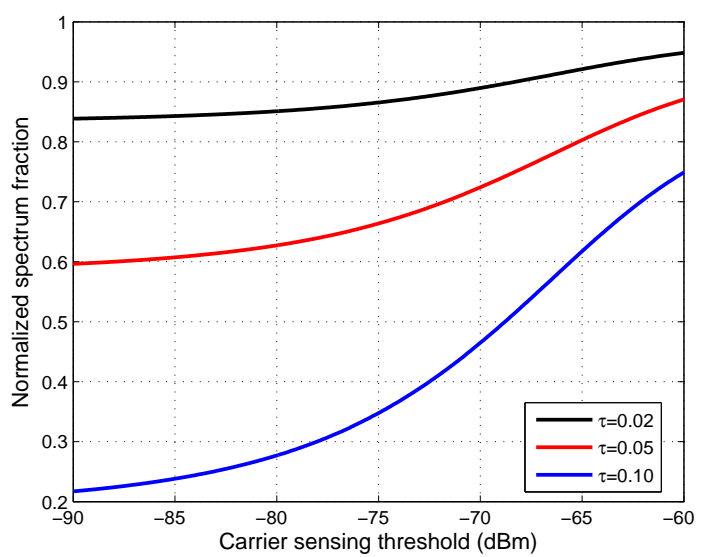

Fig. 4: Feasibility region given the target rate $\tau$ for the cellular mode and D2D user density $\lambda=10 \lambda_{b s}$.

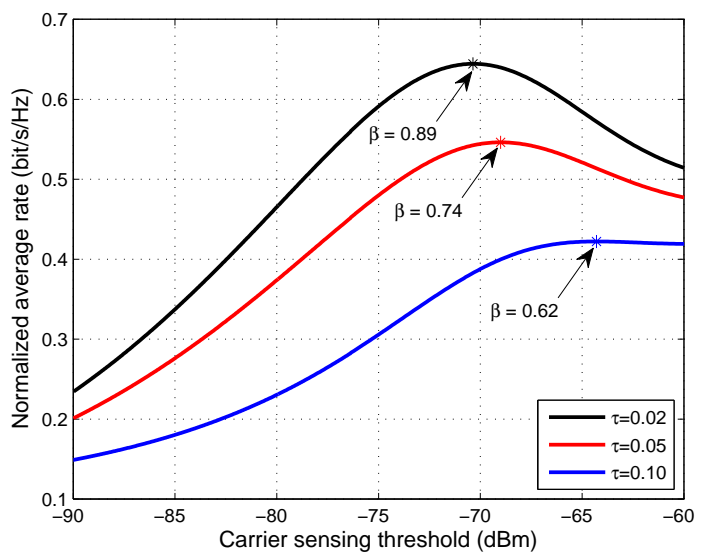

Fig. 5: Normalized rate for D2D users, D2D density $\lambda=10 \lambda_{b s}$.

In Fig. 5 we depict the rate for D2D users $Q_{d}$ as a function of the CS threshold. Note that the CS thresholds are associated with the spectrum allocation factors on the border of the feasibility region, see Fig. 4. For a low threshold the interference among D2D users is low but the associated bandwidth $\beta$ is low too. The allocated bandwidth dominates the rate in the D2D mode and through that the overall D2D rate is kept low. However, increasing the allocated bandwidth $\beta$ beyond certain point has adverse effects. Due to the associated high CS threshold, the D2D self-interference starts reducing the rate in $\mathrm{D} 2 \mathrm{D}$ mode. As a result, one can find an optimal point i.e. spectrum partition factor $\beta$ and $\mathrm{CS}$ threshold where 
the D2D user rate is maximized. As expected, the optimal point depends on the cellular rate constraint.

In Fig. 6 and Fig. 7 we depict the optimal spectrum partition factors and CS thresholds respectively for different D2D user densities. As the D2D user density increases, the number of D2D users operating in cellular mode increases too. To meet the cellular rate constraint, the reduction of available time resources per user in the cellular mode can be compensated by reserving less bandwidth for D2D mode and by setting higher CS threshold.

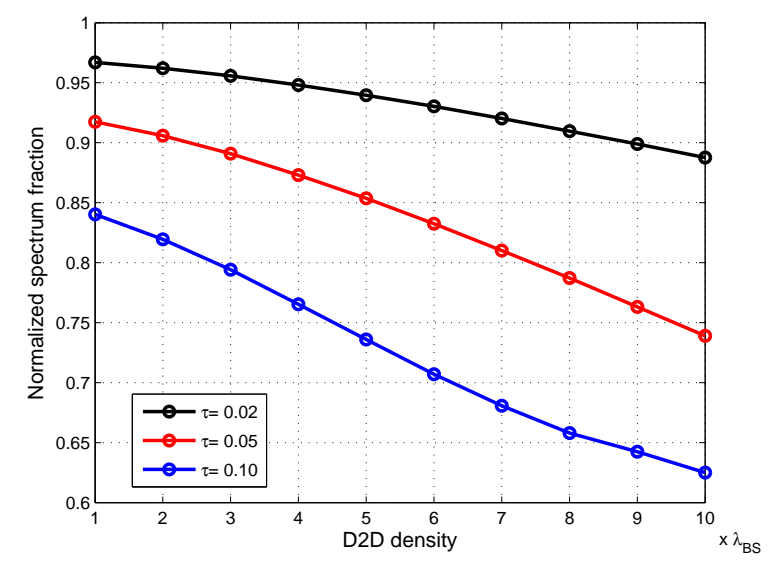

Fig. 6: Spectrum fraction $\beta$ for different D2D user densities.

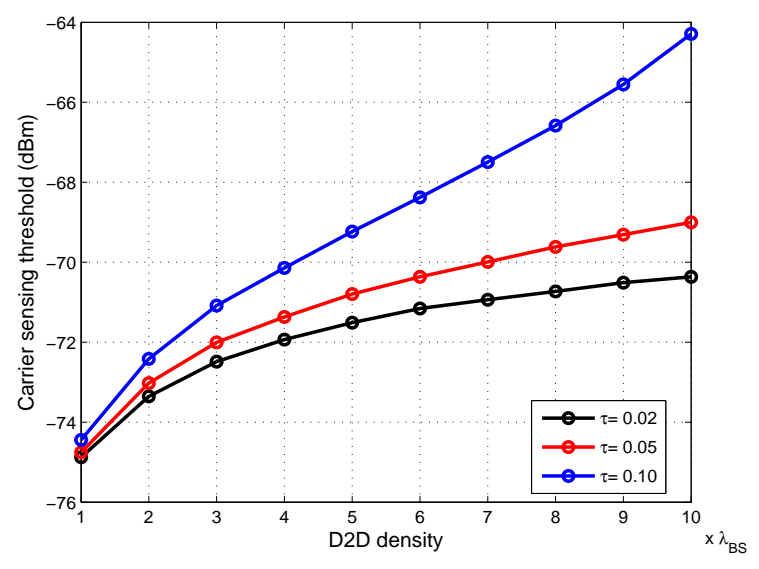

Fig. 7: CS threshold for different D2D user densities.

Finally, Fig. 8 shows the sum rate gain achieved by a cellular system enabling D2D communication in comparison with a conventional cellular system where all transmissions use the base station as a relay. The gain is computed as follows:

$$
\frac{\lambda_{c} \cdot Q_{c}+\lambda \cdot Q_{d}}{\left(\lambda_{c}+\lambda\right) \cdot R_{c}}
$$

where for the conventional system the cellular rate, $R_{c}$, should be evaluated after setting in equation (6) the D2D spectrum allocation factor $\beta=0$.
When the D2D user density becomes high, the spectrum allocation factor $\beta$ decreases and at the same time, the density of users in D2D mode increases, see Fig. 6 and Fig. 7. Due to these reasons, the rate of users in D2D mode, $R_{d}$, decreases and subsequently the overall benefit of localized communication decreases too. However, for all considered D2D user densities, significant gain has been computed.

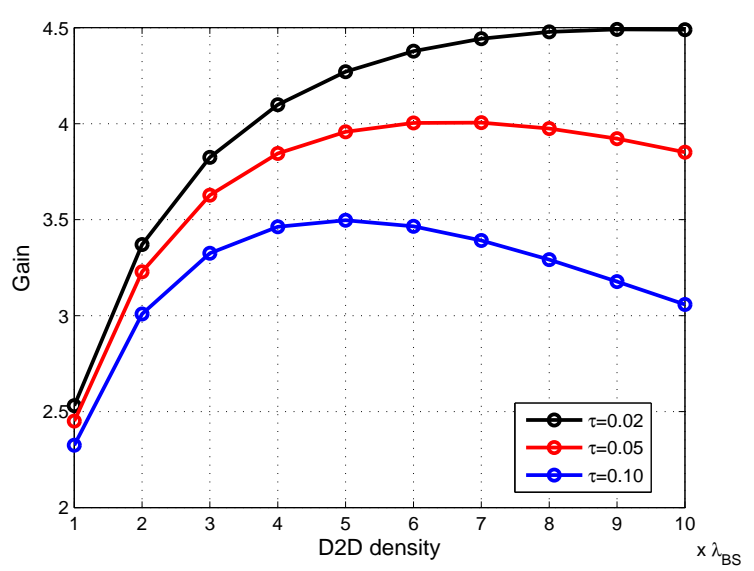

Fig. 8: Gain in comparison with a cellular system without D2D mode functionality.

\section{Conclusions}

In this paper we proposed a mechanism to allocate spectrum for in-band overlay D2D communication. The mechanism has a distributed nature eliminating communication signalling overhead between D2D users and their home BSs. We have ended up with relative high fractions of cellular bandwidth allocated for D2D. This is due to the fact that the objective function in our optimization considers simply the D2D user rate while the cellular user rate appears only as an optimization constraint. As potential directions for future work one may consider how the spectrum allocation behaves when the objective function incorporates performance aspects both for cellular and D2D users. Also, adjacent channel interference issues between the cellular and D2D mode would have an impact on the optimal carrier sensing threshold and spectrum allocation factor. Finally, extending the proposed mechanism to incorporate multi-operator D2D communication aspects would increase its applicability.

\section{APPENDIX}

\section{A. Derivation of equation (4)}

The coverage probability for D2D mode is:

$$
\begin{aligned}
\mathcal{P}_{d} & =\mathbb{P}\left\{\operatorname{SINR}>\gamma_{d}\right\}=\mathbb{P}\left\{\frac{P_{d} h l_{d}(d)}{\beta \sigma_{d}^{2}+I_{d}}>\gamma_{d}\right\} \\
& =\mathbb{P}\left\{h>\frac{\gamma_{d}\left(\beta \sigma_{d}^{2}+I_{d}\right)}{P_{d} l_{d}(d)}\right\}=\mathbb{E}_{I_{d}}^{! o}\left\{e^{-\frac{\gamma_{d}\left(\beta \sigma_{d}^{2}+I_{d}\right)}{P_{d^{l} d}(d)}}\right\} \\
& =e^{-\frac{\gamma_{d}^{\beta \sigma_{d}^{2}}}{P_{d^{l} d}(d)}} \mathcal{L}_{I_{d}}\left(\frac{\gamma_{d}}{P_{d} l_{d}(d)}\right)
\end{aligned}
$$


where $\mathcal{L}_{I_{d}}\left(\frac{\gamma_{d}}{P_{d} l_{d}(d)}\right)$ is the Laplace transform (LT) of aggregate interference at the $\mathrm{D} 2 \mathrm{D}$ receiver evaluated at $s=\frac{\gamma_{d}}{P_{d} l_{d}(d)}$.

$$
\begin{aligned}
\mathcal{L}_{I_{r}} & =\mathbb{E}_{\Phi_{d}, h}^{! o}\left\{e^{-\frac{\sum_{x} P_{d} h_{x} l_{d}\left(r_{x}\right)}{P_{d} l_{d}(d) / \gamma_{d}}}\right\}=\mathbb{E}_{\Phi_{d}, h}^{! o}\left\{\prod_{x} e^{-\frac{h_{x} l_{d}\left(r_{x}\right)}{l_{d}(d) / \gamma_{d}}}\right\} \\
& =\mathbb{E}_{\Phi_{d}\left(S_{1}\right), h}^{! o}\left\{\prod_{x} e^{-\frac{h_{x} l_{d}\left(r_{x}\right)}{l_{d}(d) / \gamma_{d}}}\right\} \cdot \mathbb{E}_{\Phi_{d}\left(S_{2}\right), h}^{!^{\prime}}\left\{\prod_{x} e^{-\frac{h_{x} l_{d}\left(r_{x}\right)}{l_{d}(d) / \gamma_{d}}}\right\} \\
& =\mathbb{E}_{\Phi_{d}\left(S_{1}\right)}^{! o}\left\{\prod_{x} \frac{1}{1+\frac{\gamma_{d} l_{d}\left(r_{x}\right)}{l_{d}(d)}}\right\} \cdot \mathbb{E}_{\Phi_{d}\left(S_{2}\right)}^{! o}\left\{\prod_{x} \frac{1}{1+\frac{\gamma_{d} l_{d}\left(r_{x}\right)}{l_{d}(d)}}\right\}
\end{aligned}
$$

where $S_{1}=\{(r, \theta): 0 \leq \theta \leq 2 \pi, r \geq 2 \delta\}, S_{2}=\{(r, \theta): 0 \leq$ $\theta \leq 2 \pi, \delta<r<2 \delta\}$ and $r_{x}$ is the distance between the $x$-th $\mathrm{D} 2 \mathrm{D}$ transmitter and the $\mathrm{D} 2 \mathrm{D}$ receiver under consideration, see Fig. 9.

While the point process $\Phi_{d}\left(S_{1}\right)$ is Poisson with density $\lambda_{d}$, the point process $\Phi_{d}\left(S_{2}\right)$ is not due to the positive correlation of MPP type II at distances less than $2 \delta$. However, the mean interference received from the area $S_{2}$ can be upper bounded by a PPP with density $c \lambda_{d}$ [12]. Based on that, we make the assumption that the PGFL of an MPP type II in the area $S_{2}$ can be lower bounded by the PGFL of a PPP with density $c \lambda_{d}$ and the desired result is obtained.

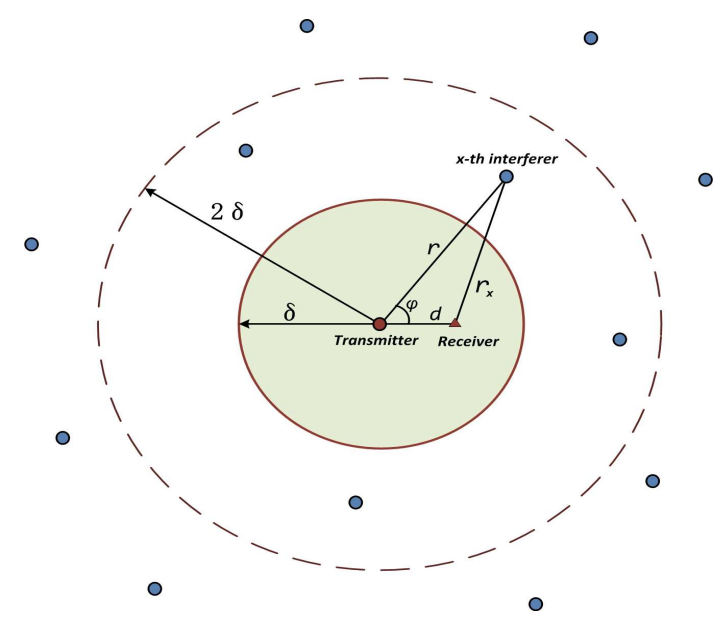

Fig. 9: Generated interference at D2D receiver due to transmissions in D2D mode.

\section{B. Proof of Lemma 1}

Unlike the D2D case where the useful link distance is fixed and equal to $d$, the distance between a cellular user and its home BS follows a Rayleigh distribution $h_{R}(r)$. Following similar steps as in the Appendix A we obtain

$$
\mathcal{P}_{c}=\int_{0}^{\infty} h_{R}(r) \cdot e^{-\frac{\gamma_{c}(1-\beta) \sigma_{c}^{2}}{P_{c} l_{c}(r)}} \cdot \mathcal{L}_{I_{c}}\left(\frac{\gamma_{c}}{P_{c} l_{c}(r)}\right) d r
$$

where $I_{c}$ is the aggregate out-of-cell interference from uplink cellular transmissions. The distribution of the random variable
$I_{c}$ is characterized in terms of LT which is given by

$$
\begin{aligned}
\mathcal{L}_{I_{c}} & =\mathbb{E}_{\Phi_{c, a}^{\prime}}^{\prime o}, h\left\{e^{-\frac{\gamma_{c}}{P_{c} l_{c}(r)} \sum_{y} P_{c} h_{y} l_{c}\left(r_{y}\right)}\right\} \\
& \left.\stackrel{(p 1)}{=} \mathbb{E}_{\Phi_{c, a}^{\prime}, o}^{\prime}, h \prod_{y} e^{-\frac{\gamma_{c} h_{y} l_{c}\left(r_{y}\right)}{l_{c}(r)}}\right\}=\mathbb{E}_{\Phi_{c, a}^{\prime}}^{\prime o}\left\{\prod_{y} \frac{1}{1+\frac{\gamma_{c} l_{c}\left(r_{y}\right)}{l_{c}(r)}}\right\} \\
& \stackrel{(p 2)}{=} e^{-2 \pi \alpha \lambda_{b s} \int_{r}^{\infty} \frac{\gamma_{c} l_{c}\left(r_{y}\right) / l_{c}(r)}{1+\gamma_{c} l_{c}\left(r_{y}\right) / l_{c}(r)} r_{y} d r_{y}} \\
& \stackrel{(p 3)}{=} e^{-2 \pi \alpha \lambda_{b s} \frac{r^{2} \gamma_{c}}{a-2} 2 F_{1}\left(1, \frac{a-2}{a}, 2-\frac{2}{a},-\gamma_{c}\right)}
\end{aligned}
$$

where $(p 1)$ follows from the i.i.d. distribution of the fading $h_{y},(p 2)$ follows from the PFGL of PPP $\Phi_{c, a}^{\prime}$ and the fact that the fading $h_{y}$ follows an exponential distribution with mean equal to unity, and ( $p 3)$ follows from the following relationship $\int_{r}^{\infty} \frac{u}{u+x^{a}} x d x=\frac{u r^{2-a}}{a-2}{ }_{2} F_{1}\left(1, \frac{a-2}{a}, 2-\frac{2}{a},-u r^{-a}\right)$.

\section{ACKNOWLEDGMENT}

This work was supported in part by the Academy-of-Finland funded project SMACIW under Grant no. 265040 and part of this work has been performed in the framework of the FP7 project ICT 317669 METIS, which is partly funded by the European Union.

\section{REFERENCES}

[1] "Initial report on horizontal topics, first results and $5 \mathrm{G}$ system concept," Document Number: ICT-317669-METIS/D6.2, available at https://www.metis2020.com/documents/deliverables/

[2] G. Fodor, E. Dahlman, G. Mildh, S. Parkvall, N. Reider, G. Mikls, and Z. Turnyi, "Design aspects of network assisted device-to-device communications," IEEE Communications Magazine, vol. 50, no. 3, pp. 170-177, Mar. 2012.

[3] K. Doppler, M. Rinne, C. Wijting, C. B. Ribeiro, and K. Hugl, "Device-to-device communication as an underlay to LTE-advanced networks," IEEE Communications Magazine, vol. 47, no. 12, pp. 4249, Dec. 2009

[4] X. Lin, J. G. Andrews and A. Ghosh, "Spectrum sharing for device-to-device communication in cellular networks," submitted to IEEE Transactions on Wireless Communications. Available at http://arxiv.org/abs/1305.4219.

[5] C.-H. Yu, K. Doppler, C. B. Ribeiro, and O. Tirkkonen, "Resource sharing optimization for device-to-device communication underlaying cellular networks," IEEE Transactions on Wireless Communications, vol. 10, no. 8, pp. 2752-2763, Aug. 2011.

[6] D. Stoyan, W. Kendall, and J. Mecke, Stochastic Geometry and Its Applications. 2nd ed. John Wiley and Sons Ltd, 1996.

[7] Z. Chen, C. Wang, X. Hong, J. Thompson, S. A. Vorobyov, X. Ge, H. Xiao, and F. Zhao, "Aggregate interference modeling in cognitive radio networks with power and contention control," IEEE Transactions on Communications, vol. 60, no. 2, pp. 456-468, Feb. 2012.

[8] B. Cho, K. Koufos, and R. Jäntti, "Interference control in cognitive wireless networks by tuning the carrier sensing threshold," in Proc. IEEE CrownCom, pp. 282-287, Jul. 2013.

[9] B. Cho, K. Koufos, and R. Jäntti, "Bounding the mean interference in Matérn type II hard-core wireless networks," IEEE Wireless Communications Letters, vol. 2, no. 5, pp. 563-566, Oct. 2013.

[10] S.-M. Yu and S.-L. Kim, "Downlink capacity and base station density in cellular networks," in Proc. IEEE WiOpt, pp. 119-124, May. 2013.

[11] 3GPP, "TR 30.03U version 3.2.0: Universal mobile telecommunications system(UMTS); Selection procedures for the choice of radio transmission technologies of the UMTS, technical report," 1998.

[12] M. Haenggi, "Mean Interference in Hard-Core Wireless Networks," IEEE Communications Letters, vol. 15, no. 8, pp. 792-794, Aug. 2011. 